\title{
Rational Approximation with a Vanishing Weight Function and with a Fixed Value at Zero
}

\author{
By Charles B. Dunham \\ In Memory of Hirondo Kuki
}

\begin{abstract}
Chebyshev approximation by ordinary rational functions with respect to a vanishing weight function is considered. A best approximation is characterized by alternation and is unique but may not exist. The problem arises in Kuki's technique for rational approximation with interpolation at zero and with Williams' interpolating rationals.
\end{abstract}

Let $[a, b]$ be a finite interval and $C[a, b]$ be the set of continuous functions on $[a, b]$. For $g \in C[a, b]$ define

$$
\|g\|=\max \{|g(x)|: a \leqslant x \leqslant b\} .
$$

Let $s$ be a continuous nonnegative function (which does not vanish identically). Let $R_{m}^{n}[a, b]$ be the family of ratios of polynomials $p$ of degree $n$ to polynomials $q$ of degree $m, q(x)>0$ for $a \leqslant x \leqslant b$. The approximation problem with weight $s$ is: given $f \in C[a, b]$ to find $r^{*} \in R_{m}^{n}[a, b]$ to minimize $\|s(f-r)\|$. Such an approximation $r^{*}$ is called best to $f$.

1. Existence of a Best Approximation. In the case $m=0$, the approximation problem is one of linear approximation, and a best approximation, therefore, exists. In the case $m \geqslant 1$, the theory of approximation by generalized rational functions, as developed by Boehm and Goldstein [6, pp. 84-89], guarantees that there is a ratio $r=$ $p / q$ such that $\|s(f-r)\|$ is minimal, but such an $r$ may have a pole in $[a, b]$.

Example. Let $[a, b]=[0,1], n=0, m=1, s(x)=x$, and

$$
\begin{aligned}
f(x) & =2, & & 0 \leqslant x \leqslant 1 / 2, \\
& =1 / x+4 \sin (6 \pi x) / x, & & 1 / 2 \leqslant x \leqslant 1 .
\end{aligned}
$$

Let $r_{0}(x)=1 / x$; then $\left\|s\left(f-r_{0}\right)\right\|=4$ and $s\left(f-r_{0}\right)$ alternates twice on $[0,1]$ with extrema at $7 / 12,9 / 12,11 / 12$. From this it follows by standard arguments in rational approximation that $r_{0}$ is the unique ratio of polynomials of degree 0 to polynomials of degree 1 which minimizes $\|s(f-r)\|$. We have $\|s(f-1 /(1 / k+x))\| \rightarrow 4$; so no best approximation in $R_{1}^{0}[0,1]$ exists.

It should be noted that Taylor and Williams [8] have related examples of nonexistence in Williams' problem. 
2. Characterization of Best Approximations. Let $\rho(0)=n+1$. Let $r \neq 0$ be a ratio of $p / q, p$ and $q$ relatively prime, degree of $p=\partial p$, degree of $q=\partial q$, and let $\rho(r)=$ $n+m+1-\min \{n-\partial p, m-\partial q\}$. From [2], which generalizes results of [1], we obtain

THEOREM. Let $r \in R_{m}^{n}[a, b]$, then $r$ is best to $f$ if and only if $s(f-r)$ alternates $\rho(r)$ times. $A$ best approximation is unique.

As this is identical to the standard alternation result, this suggests that we use the standard rational Remez algorithm with weight function $s$ to compute the best approximation. We, of course, start the algorithm with points at which $s$ does not vanish. Analysis of standard convergence results for the weighted rational Remez algorithm shows that they apply to our more general weight function.

3. Rational Approximation with a Fixed Value at Zero. Let $f(0) \neq 0$, and suppose that we wish a rational approximation taking the value $f(0)$ at 0 . Let us consider approximations of the form $f(0)+x r$. These have the property that when evaluated in a machine they will take the desired value at zero. They also have the advantage of numerical stability if $\boldsymbol{r}$ is small. Approximations of this type are frequeritly used by Kuki for the IBM 7094 library [4] and the IBM 360 library [3].

Consider the problem of choosing $r$ in $R_{m}^{n}[0, b]$ to minimize $\|w(f-f(0)-x r)\|$. We can rewrite the norm as $\|w x([(f-f(0)) / x]-r)\|$. If $(f-f(0)) / x$ is continuous at $x=0$, which is true if $f$ has a convergent Taylor series expansion about zero, this reduces to the approximation problem of the earlier sections. This technique is due to Kuki $[5$, p. 51$]$.

Let $w=1$ and

$$
\begin{aligned}
f(x) & =1+2 x, & & 0 \leqslant x \leqslant 1 / 2, \\
& =2+4 \sin (6 \pi x), & & 1 / 2 \leqslant x \leqslant 1 .
\end{aligned}
$$

When we apply Kuki's technique to $f$, we end up with the function of the example of nonexistence in Section 1. Hence, existence of a best approximation in Kuki's problem is not guaranteed unless $m=0$.

4. Interpolating Rationals. J. Williams [7] has considered the case where the function to be approximated is of the form $B(x) f(x), f(x)>0$ for $\alpha \leqslant x \leqslant \beta$, and is to be approximated in the Chebyshev sense by $B(x) / p, p$ a positive polynomial of degree $m$. Let $s(x)=|B(x)|$; then this problem of Williams is equivalent to our problem of weighted approximation of $f$ by $R_{m}^{0}[\alpha, \beta]$. As noted earlier, we can apply the standard rational Remez algorithm, making it unnecessary to develop a special program for this case of Williams' problem.

Computer Science Department University of Western Ontario London, Ontario, Canada

1. C. B. DUNHAM, "Chebyshev approximation with respect to a weight function," J.Approximation Theory, v. 2, 1969, pp. 223-232. MR 40 \#6137.

2. C. B. DUNHAM, "Chebyshev approximation with respect to a vanishing weight function," J. Approximation Theory, v. 12, 1974, pp. 305-306. 
3. IBM System/360 FORTRAN IV Library Subprograms, 5th ed., October 1968.

4. H. KUKI, Mathematical Functions, University of Chicago Computation Center Report, 1966.

5. H. KUKI \& J. ASCOLY, “FORTRAN extended-precision library," IBM Systems J., v. 10, 1971, pp. 39-61.

6. J. R. RICE, The Approximation of Functions. Vol. 2: Nonlinear and Multivariate Theory, Addison-Wesley, Reading, Mass., 1969. MR 39 \#5989.

7. J. WILLIAMS, "Numerical Chebyshev approximation by interpolating rationals," Math. Comp., v. 26, 1972, pp. 199-206.

8. G. D. TAYLOR \& J. WILLIAMS, "Existence questions for the problem of Chebyshev approximation by interpolating rationals," Math. Comp., v. 28, 1974, pp. 1097-1103. 